\title{
Integrated approach using data mining-based decision tree and object-based image analysis for high-resolution urban mapping of WorldView-2 satellite sensor data
}

\begin{abstract}
This paper integrates decision tree-based data mining (DM) and object-based image analysis (OBIA) to provide a transferable model for the detailed characterization of urban land-cover classes using WorldView-2 (WV-2) satellite images. Many articles have been published on OBIA in recent years based on DM for different applications. However, less attention has been paid to the generation of a transferable model for characterizing detailed urban land cover features. Three subsets of WV-2 images were used in this paper to generate transferable OBIA rule-sets. Many features were explored by using a DM algorithm, which created the classification rules as a decision tree (DT) structure from the first study area. The developed DT algorithm was applied to object-based classifications in the first study area. After this process, we validated the capability and transferability of the classification rules into second and third subsets. Detailed ground truth samples were collected to assess the classification results. The first, second, and third study areas achieved $88 \%, 85 \%$, and $85 \%$ overall accuracies, respectively. Results from the investigation indicate that DM was an efficient method to provide the optimal and transferable classification rules for OBIA, which accelerates the rule-sets creation stage in the OBIA classification domain.
\end{abstract}

Keyword: Integrated approach; Data mining-based decision tree; Object-based image analysis; High-resolution urban mapping; WorldView-2 satellite sensor data 